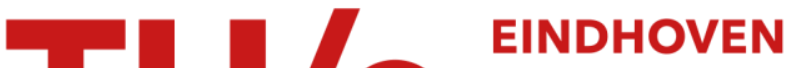

\section{From optimal to real-time control of a mechanical hybrid powertrain}

Citation for published version (APA):

van Berkel, K., Titulaer, R. H. M., Hofman, T., Vroemen, B., \& Steinbuch, M. (2015). From optimal to real-time control of a mechanical hybrid powertrain. IEEE Transactions on Control Systems Technology, 23(2), 670-678. [6862868]. https://doi.org/10.1109/TCST.2014.2334476

\section{Document license:}

TAVERNE

DOI:

10.1109/TCST.2014.2334476

Document status and date:

Published: 01/03/2015

\section{Document Version:}

Publisher's PDF, also known as Version of Record (includes final page, issue and volume numbers)

\section{Please check the document version of this publication:}

- A submitted manuscript is the version of the article upon submission and before peer-review. There can be important differences between the submitted version and the official published version of record. People interested in the research are advised to contact the author for the final version of the publication, or visit the $\mathrm{DOI}$ to the publisher's website.

- The final author version and the galley proof are versions of the publication after peer review.

- The final published version features the final layout of the paper including the volume, issue and page numbers.

Link to publication

\section{General rights}

Copyright and moral rights for the publications made accessible in the public portal are retained by the authors and/or other copyright owners and it is a condition of accessing publications that users recognise and abide by the legal requirements associated with these rights.

- Users may download and print one copy of any publication from the public portal for the purpose of private study or research.

- You may not further distribute the material or use it for any profit-making activity or commercial gain

- You may freely distribute the URL identifying the publication in the public portal.

If the publication is distributed under the terms of Article 25fa of the Dutch Copyright Act, indicated by the "Taverne" license above, please follow below link for the End User Agreement:

www.tue.nl/taverne

Take down policy

If you believe that this document breaches copyright please contact us at:

openaccess@tue.nl

providing details and we will investigate your claim. 


\title{
From Optimal to Real-Time Control of a Mechanical Hybrid Powertrain
}

\author{
Koos van Berkel, Member, IEEE, Roel Titulaer, Theo Hofman, Bas Vroemen, \\ and Maarten Steinbuch, Senior Member, IEEE
}

\begin{abstract}
This brief presents the design of an energy controller for a mechanical hybrid powertrain, which is suitable for implementation in real-time hardware. The mechanical hybrid powertrain uses a compact flywheel module to add hybrid functionalities to a conventional powertrain that consists of an internal combustion engine and a continuously variable transmission. The control objective is to minimize the overall fuel consumption for a given driving cycle. The design approach follows a generic framework to: 1) solve the optimization problem using optimal control; 2) make the optimal controller causal using a prediction of the future driving conditions; and 3) make the causal controller robust by tuning of one key calibration parameter. The highly constrained optimization problem is solved with dynamic programming. The future driving conditions are predicted using a model that smoothly approximates statistical data, and implemented in the receding model predictive control framework. The controller is made tunable by rule extraction from the model predictive controller, based on physical understanding of the system. The resulting real-time controller is transparent, causal, and robust, where the latter is shown by simulations for various driving cycles and start conditions.
\end{abstract}

Index Terms-Energy management, flywheel, hybrid powertrain, optimal control, real-time control.

\section{INTRODUCTION}

$\mathbf{H}$ YBRID powertrains have the potential to improve the fuel economy of passenger vehicles significantly by adding a secondary power source to the internal combustion engine. A low-cost alternative to the state-of-the-art hybrid electric powertrains is a mechanical hybrid powertrain, which uses a compact flywheel system for kinetic energy storage and standard mechanical components such as a Continuously Variable Transmission (CVT) and clutches for power transmission [1]. The powertrain topology is schematically shown in Fig. 1. The fuel-saving benefits can be attributed to three functionalities, which are: 1) recuperation of brake energy for later use; 2) elimination of inefficient part-load operation of

Manuscript received October 27, 2013; revised March 25, 2014; accepted June 22, 2014. Date of publication July 23, 2014; date of current version February 11, 2015. Manuscript received in final form on June 26, 2014. This work was supported by the Pieken in de Delta Program through the mecHybrid Project, in part by the Dutch Ministry of Economic Affairs, Province NoordBrabant, and in part by Samenwerkingsverband Regio Eindhoven, Eindhoven, The Netherlands. Recommended by Associate Editor S. Di Cairano.

K. van Berkel was with the Control Systems Technology Group, Department of Mechanical Engineering, Eindhoven University of Technology, Eindhoven $5600 \mathrm{MB}$, The Netherlands. He is now with the Research Mechatronics and Control Department, ASML, 5500 AH Veldhoven, The Netherlands (e-mail: k.v.berkel@gmail.com).

R. Titulaer and B. Vroemen are with Punch Powertrain, Eindhoven 5653 LD, The Netherlands (e-mail: roel.titulaer@punchpowertrain.com; bas.vroemen@punchpowertrain.com).

T. Hofman and M. Steinbuch are with the Department of Mechanical Engineering, Eindhoven University of Technology, Eindhoven 5600 MB, The Netherlands (e-mail: t.hofman@tue.nl; m.steinbuch@tue.nl).

Digital Object Identifier 10.1109/TCST.2014.2334476

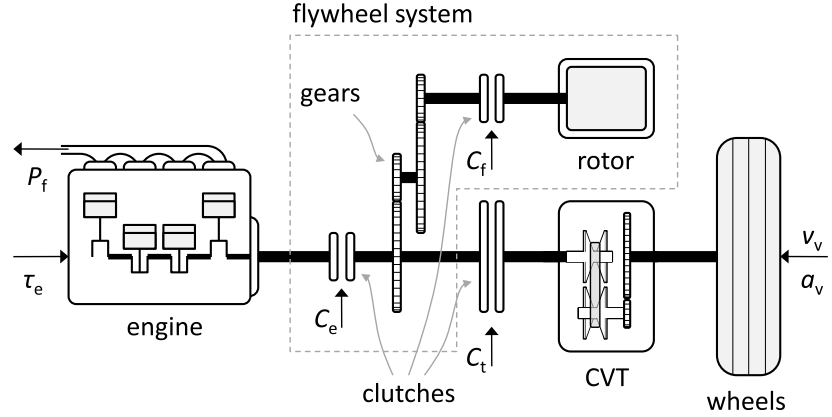

Fig. 1. Schematic representation of the mechanical hybrid powertrain.

the engine; and 3) engine shutoff during vehicle standstill. A possible benefit of engine downsizing is not considered owing to the limited energy storage capacity of the flywheel system [2].

To make full use of these functionalities, a controller is required, which controls on system level the power distribution between the engine, flywheel system, and vehicle. The main objective of this controller is to minimize the overall fuel consumption for a given driving cycle, subject to the constraints imposed by the powertrain dynamics, physical operating limits of the components, and driving comfort requirements. This energy controller mainly focuses on the relatively slow energy buffer dynamics, whereas it serves as a setpoint for a torque controller that focuses on the much faster torque dynamics, e.g., during clutch engagement [3]. In order to keep the cost of the hybrid system potentially low, this energy controller may not rely on prediction systems based on inputs given by the driver (e.g., navigation), or sensors (e.g., global positioning system, telemetry radar). The controller may only use limited computation and memory resources to be suitable for implementation in real-time hardware. Furthermore, the controller must be tunable to increase its robustness against measurement and prediction uncertainties. To enhance in-vehicle calibration, the controller design must be transparant to understand the impact of each calibration parameter, whereas the number of calibration parameters needs to be small [4].

\section{A. Real-Time Energy Controller Design}

The design of such a real-time energy controller is widely studied in the literature, resulting in several approaches as described in [5]. Promising results are obtained with systematic approaches based on optimal control. A general approach that is often followed can be captured by three design steps.

1) Solve the deterministic optimal control problem offline, to obtain an optimization tool, which is useful: a) to 
reduce the optimization problem by eliminating insignificant states and control variables [6]; b) to optimize the topology and component sizes of the hybrid powertrain [2]; and c) as a performance benchmark for the real-time controller.

2) Make the controller causal by solving the optimal control problem for a prediction of the future driving conditions. The prediction horizon must be selected as a tradeoff between the prediction accuracy and the time scale of the relevant energy dynamics.

3) Increase the controller robustness against modeling and prediction uncertainties, by the abstraction of a tunable controller, for example, by introducing penalty functions [7], [8], rule functions [9], or (feedback) control parameters [10]. Then, safety margins can be introduced to avoid state constraint violation and to avoid unnecessary control decisions such as chattering between discrete states.

Steps 2 and 3 can be (implicitly) combined in one design step [10]; however, explicit separation of these steps enables to understand the impact of uncertainties in prediction and in abstraction separately. Step 2 can also be omitted, as rules may be directly extracted from the optimal control results (Step 1) for a given set of driving cycles [9]. In practice, however, this gives two problems that scale with the number of states: 1) for a limited set of driving cycles and initial states, the optimal controller usually does not cover all possible driving and powertrain states and 2) for each combination of driving and powertrain states, the optimal control decision still depends on the selected (part of) the driving cycle, hence is not unique.

In Step 1, (semi) analytical approaches based on the minimum principle are often proposed for relatively simple hybrid electric powertrains [11], [12]. Such approaches are typically not suitable for mechanical hybrid powertrains that contain: 1) switched dynamics due to the use of clutches; 2) active state constraints due to use a relatively small energy storage capacity and mechanical connections; and 3) nonconvex control constraints to avoid uncomfortable driving mode switches. Optimal control is usually not used for this class of mechanical hybrid powertrains ${ }^{1}$ [1]. However, numerical optimization methods that can handle such characteristics, such as Dynamic Programming (DP), can be used with the same design approach as will be explained next.

In Step 2, a causal controller can be obtained using Stochastic DP (SDP), which solves the optimal control problem for a family of stochastic driving cycles described by statistical probability distributions [13], [14]. Because the method examines all possible stochastic driving cycles, the overall (offline) computation time may become infeasible for design purposes (e.g., longer than one day), hence SDP is often implemented with an efficient numerical solver such as a linear or quadratic programming [15]. The underlying model, however, may be compromised to fit in the framework. Alternatively, the optimization problem may be substantially reduced by minimizing only the cost of an expected driving

\footnotetext{
${ }^{1}$ An extensive list of mechanical hybrid powertrains can be found in [2]
}

cycle, instead of the expected cost for all possible stochastic driving cycles. Then, the expected driving cycle serves as a prediction in a deterministic Model Predictive Control (MPC) framework [16]. The solution is inherently suboptimal, but due to the reduced computation time, a more versatile solver (i.e., DP) can be used with a more realistic model. As a result, more computation time is spend on modeling details of the powertrain, whereas less computation time is spend on the rather uncertain (i.e., driver- and traffic-specific) driving conditions. The explicit controller obtained with MPC (as well as with SDP) is described by a (multi-dimensional) matrix, of which each element describes the optimal control variable (i.e., for the prediction) as a function of the quantized powertrain and driving states. This precomputed controller can be very fast once it is implemented, though in-vehicle calibration remains cumbersome and time consuming, as each adjustment in one of the calibration parameters (e.g., weight of a mixed cost function) requires a new, offline computation. The control matrix is typically characterized by control regions, where neighboring elements have the same value. Prediction uncertainties are reflected in uncertain shapes of these regions, whereas nonsmooth irregularities in these shapes may arise owing to quantization of the system variables and the use of a finite prediction horizon.

In Step 3, these (numerical) problems can be avoided by extracting rule functions that give a smooth approximation of the control region boundaries [17]. Consequently, these control region boundaries can be tuned without recomputing the control matrices. When the rule extraction is based on physical understandings of the system, insights are gained in the implicit decision process of the numerical controller, which is very useful to identify the key calibration parameters that increase the robustness against modeling and prediction uncertainties. The abstraction process follows the philosophy of grounded theory [18], as in-depth understanding of the system is exploited to explain the causal controller data, whereas system understanding is also generated from the analysis of this data.

\section{B. Main Contributions and Outline}

This brief contributes with a systematic design of a realtime controller for the mechanical hybrid powertrain, following a numerical optimal control-based design approach using DP, MPC, and rule extraction. The controller is not implemented in real-time hardware, yet the two key challenges regarding causality and tunability will be addressed. The future driving conditions are predicted with a deterministic expectation model that is based on driving cycle statistics and approximated with a smooth functional fit. Physically interpretable rules are extracted to provide insights in the decision process and to enable calibration for robustness against uncertainty in the predicted driving conditions. Although the extraction of physically interpretable rules is a powertrain-specific challenge, the presented numerical design approach is suitable for a wide class of hybrid powertrains with relatively complex characteristics, such as switched dynamics (i.e., topology with multiple driving modes), active 
state constraints (i.e., downsized components), and nonconvex control constraints (i.e., to avoid uncomfortable control actions).

The outline of this brief is as follows. Section II describes the modeling of the mechanical hybrid powertrain. Section III describes the design of the driving cycle model. Section IV describes the real-time controller design, for which the simulation results are discussed in Section V. Section VI summarizes the results and conclusions.

\section{Mechanical Hybrid Powertrain Modeling}

The mechanical hybrid powertrain consists of a fourcylinder $76-\mathrm{kW}$ gasoline internal combustion engine, a vacuum-placed 150-kJ flywheel system, three clutches, a conventional pushbelt CVT, and a compact vehicle including two passengers with a total mass of $1120 \mathrm{~kg}$ (as schematically shown in Fig. 1).

\section{A. Dynamics}

The modeling of the motion dynamics is extensively described in [1] and shortly summarized below. The longitudinal dynamics are described for the most relevant (largest) inertias, that is, that of the flywheel and the vehicle. The transmission clutch $\left(C_{t}\right)$ is used to accelerate the vehicle or flywheel from standstill. The engine clutch $\left(C_{e}\right)$ and the flywheel clutch $\left(C_{f}\right)$ are only designed to select driving modes by mechanical (dis-)engagement of powertrain parts (i.e., with low power dissipation). There are three relevant driving modes.

1) Flywheel Driving: The flywheel propels or brakes the vehicle while the engine is disengaged and shutoff $(\phi=1)$.

2) Hybrid Driving: The engine and flywheel propel the vehicle, where the contribution of each power source is determined by the relative power split $(\phi=2)$.

3) Engine Driving: During propulsion, the engine propels the vehicle while the flywheel is disengaged and coasting, whereas during braking, the flywheel brakes the vehicle while the engine is disengaged and idling $(\phi=3)$.

The power dissipation in the clutches are based on first principle models described in [1], whereas that of the CVT and flywheel system are based on semi-empirical models described in [19]. The motion dynamics is extended with the main thermodynamics in the powertrain as described in [6] to include the significant impact of cold start conditions on the power dissipation in the powertrain. The combined dynamics can be described in a discrete time format using index $k$ and a fixed time step of $\Delta t=1 \mathrm{~s}$ by

$$
x(k+1)=x(k)+f(k, x(k), u(k), w(k)) \Delta t .
$$

The state vector $x(k)$ contains three states, which are: 1$)$ the kinetic energy in the rotor of the flywheel system (or, flywheel energy) $E_{r}(k)$ to describe the constrained energy dynamics; 2 ) the previous driving mode $\phi_{\text {pre }}(k)=\phi(k-1)$ to describe costs and constraints on driving mode switches; and 3) the powertrain temperature $\vartheta_{p}(k)$ to describe the temperaturedependent power dissipation in the engine and transmission, which can be influenced by shortening the heating time. The control variable vector $u(k)$ contains the current driving mode $\phi(k)$ and the relative power split $\sigma(k)$ in the hybrid driving mode, which implicitly control the clutches states and the speed ratio of the CVT, respectively. The external state vector $w(k)$ contains the vehicle velocity $v_{v}(k)$ and acceleration $a_{v}(k)$, prescribed by the driving cycle or predicted by a driving cycle model.

\section{B. Constraints}

The dynamics are constrained by state constraints based on physical operation limits of the components, and control constraints to guarantee a high driving comfort level.

1) State Constraints: The engine speed $\omega_{e}$ and engine torque $\tau_{e}$ are constrained by

$$
\begin{aligned}
\omega_{e} & \in\left\{0, \omega_{\text {idle }},\left[\underline{\omega}_{e}, \bar{\omega}_{e}\right]\right\} \\
\tau_{e} & \in\left[0, \bar{\tau}_{e}\left(\omega_{e}\right)\right] .
\end{aligned}
$$

The engine is either shutoff $\left(\omega_{e}=0\right)$, idling without generating power (i.e., $\omega_{e}=\omega_{\text {idle }}$ ), or running (i.e., $\omega_{e} \in\left[\underline{\omega}_{e}, \bar{\omega}_{e}\right]$ ). Physically, the engine can generate a negative torque by its friction, but that is not relevant for the controller design. The maximum engine torque is an empirical function of its rotational speed. The CVT is described by its rotational speeds $\omega$ and torques $\tau$ on both sides, that is, the primary (or, engine) side indicated by subscript $p$ and the secondary (or, drive shaft) side indicated by the subscript $s$. The speeds and torques have physical limitations, which are not critical for the controller design, except for the speed ratio $r_{v}=\omega_{s} / \omega_{p}$

$$
r_{v} \in\left[\underline{r}_{v}, \bar{r}_{v}\right] .
$$

The flywheel system is described by the flywheel energy, which is constrained by

$$
E_{r} \in\left[0, \bar{E}_{r}\right] .
$$

After the initialization of the flywheel system, the flywheel energy should exceed a lower bound $E_{r}>E_{r}>0$ for effective utilization, as will be explained next.

2) Control Constraints: The powertrain response must be fast and smooth [3] with an acceptable consistency of the engine noise. To guarantee this level of driving comfort, some dynamics must be avoided under certain conditions $(x, w)$ by constraining the control space. A state-dependent control space generally limits the feasible state space to a nonconvex subset of the unconstrained state space. An example of such a nonconvex control constraint is the irreversible driving mode switch from hybrid driving $\phi=2$ (or, flywheel driving $\phi=1$ ) to engine driving $\phi=3$. Disengagement of the flywheel clutch $\left(C_{f}\right)$ is always possible (i.e., $\phi_{\text {pre }}=2 \wedge \phi=3$ ), but the engagement (i.e., $\phi_{\text {pre }}=3 \wedge \phi=2$ ) is only allowed when no torque is transmitted as the clutch is not designed to dissipate high powers, for example, when using the disc brakes to decelerate. The second control constraint guarantees a fast torque generation of the engine after flywheel driving, for example, to handle change-of-mind actions of the driver. This requires a minimum effective flywheel speed, or implicitly a minimum effective flywheel energy $\underline{E}_{r}$, in order to crank the engine to 
the minimum engine speed $\underline{\omega}_{e}$ cf. (2), so $E_{r} \geq \underline{E}_{r}$ must hold in the flywheel driving mode $(\phi=1)$. The same constraint holds for the hybrid driving mode $(\phi=2)$, to generate sufficient torque for vehicle propulsion. The third control constraint is related to the engine noise, as its frequency rises when energizing the flywheel during hybrid driving $(\phi=2)$, which may be counterintuitive to the driver when driving at constant velocity. For an acceptable consistency of the engine noise, hybrid driving is avoided when no propulsion torque is required $\tau_{p} \leq \tau_{0}$ to keep the noise level low, where $\tau_{0}$ denotes a threshold close to zero, and outside urban areas $v_{v}>v_{u}$ to limit engine noise variations that result from duty-cycle switching between hybrid and flywheel driving. These constraints are summarized in the driving mode control space

$\phi \in \begin{cases}3 & \text { if } E_{r}<\underline{E}_{r} \vee\left(\phi_{\text {pre }} \neq 3 \wedge \tau_{p}>\tau_{0}\right) \\ \{1,3\} & \text { else if } \tau_{p} \leq \tau_{0} \vee\left(\phi_{\text {pre }} \neq 2 \wedge v_{v}>v_{u}\right) \\ \{1,2,3\} & \text { else. }\end{cases}$

\section{DRIVING CyCle Modeling}

A deterministic driving cycle model is designed based on the statistical expectation of a set of driving cycles.

\section{A. Driving Cycles}

Six representative driving cycles are selected: the New European Driving Cycle (NEDC), the Japan Cycle '08 (JC08), the Federal Test Procedure '75 (FTP75), the low, medium, and high parts of the Worldwide harmonized Light vehicles Test Procedure (WLTP), the urban part of the Common Artemis Driving Cycle (CADC), and the sportive Eindhoven driving cycle (EHV). The JC08, NEDC, and FTP75 represent certified driving cycles, whereas the WLTP, CADC, and EHV represent real-world driving behavior measured in, respectively, worldwide industrialized countries, Europe, and The Netherlands. Because the flywheel system is aimed for urban use, highway parts of WLTP and CADC are not considered.

\section{B. Dynamics and Constraints}

The driving cycles describe the vehicle velocity $v_{v}$ and vehicle acceleration $a_{v}$ as a function of discrete time $k$. The dynamics of these driving conditions can be described by a second-order model as a function of the jerk $j_{v}$

$$
\begin{aligned}
& v_{v}(k+1)=v_{v}(k)+a_{v}(k) \Delta t \\
& a_{v}(k+1)=a_{v}(k)+j_{v}(k) \Delta t .
\end{aligned}
$$

The states are constrained by the physical limitations of the vehicle, which depends on the driving conditions. These constraints are derived from the given set of driving cycles, resulting in a velocity range $v_{v} \in$ $\left[\underline{v}_{v}, \bar{v}_{v}\right]$ and a velocity-dependent acceleration range $a_{v} \in$ $\left[\underline{a}, v\left(v_{v}\right), \bar{a}_{v}\left(v_{v}\right)\right]$ that is well approximated by a fourth-order polynomial function, using the coefficients $c_{\mathrm{cs}}$ as listed in Table I

$$
\begin{aligned}
& \bar{a}_{v}\left(v_{v}\right)=c_{\mathrm{cs}}(1)+c_{\mathrm{cs}}(2) v_{v}+c_{\mathrm{cs}}(3) v_{v}^{2}+c_{\mathrm{cs}}(4) v_{v}^{3}+c_{\mathrm{cs}}(5) v_{v}^{4} \\
& \underline{a}_{v}\left(v_{v}\right)=-\bar{a}_{v}\left(v_{v}\right) .
\end{aligned}
$$

TABLE I

Driving CyCle Model CoefFicients

\begin{tabular}{l}
$c_{\mathrm{cs}}=[1200,-375,62.3,-6.28,222,-23.3,193,-244] \cdot 10^{-3}$ \\
$c_{\mathrm{dc}}=[1580,278,-29.7,0.901,-0.00877] \cdot 10^{-3}$ \\
\hline
\end{tabular}

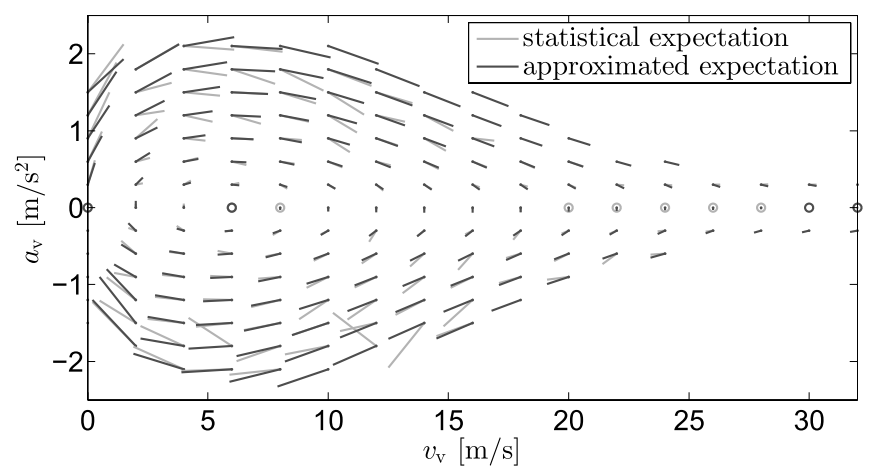

Fig. 2. Phase portraits of the driving cycle dynamics based on statistical expectation and a polynomial approximation. The circles denote local equilibria, for which $a_{v} \approx 0 \mathrm{~m} / \mathrm{s}^{2}$ and $j_{v} \approx 0 \mathrm{~m} / \mathrm{s}^{3}$ holds.

\section{Prediction}

The jerk $j_{v}$ can be predicted based on present information $\left(v_{v}, a_{v}\right)$. Using the set of driving cycles, the statistical expectation value of the jerk can be (approximately) extracted as a function of the quantized velocity and acceleration. Note that this expectation value can be considered as the average of a statistical distribution used for stochastic driving cycle models [13], [14], hence it requires substantially less samples (i.e., driving cycles) to obtain reliable statistics. The resulting phase portrait is shown in Fig. 2, where the lines denote, in clockwise direction around $v_{v}=6$ and $a_{v}=0$, trajectories of one time step (1 s) in the state space, described by the prediction model. The circles denote local equilibria implying (approximately) constant velocities. The phase portrait shows in general smooth dynamics, except for local discontinuities (i.e., crossing lines) owing to limited samples (i.e., «50). For controller design purposes, however, a smooth prediction is required for a consistent powertrain response.

The discontinuities can be smoothed by approximating the expected jerk with a semi-empirical model function. The model function describes the main dynamical characteristics, such as the symmetry around the zero acceleration axis, a high positive jerk at low velocities, no jerk around $v_{v} \approx 6 \mathrm{~m} / \mathrm{s}$, and a negative jerk that slowly attenuates at higher velocities. A suitable model function is found to be

$$
\begin{aligned}
\hat{j}_{v}\left(v_{v}, a_{v}\right)= & \left(c_{\mathrm{dc}}(1)+c_{\mathrm{dc}}(2) v_{v}+c_{\mathrm{dc}}(3) v_{v}^{2}+c_{\mathrm{dc}}(4) v_{v}^{3}\right) \\
& \cdots\left(c_{\mathrm{dc}}(5)+c_{\mathrm{dc}}(6) a_{v}+c_{\mathrm{dc}}(7) a_{v}^{2}\right) e^{c_{\mathrm{dc}}(8) v_{v}} .
\end{aligned}
$$

The coefficients $c_{\mathrm{dc}}$ (as well as for $c_{\mathrm{cs}}$ ) are identified using a standard least-square fitting method and are listed in Table I. The resulting phase portraits in Fig. 2 show that the empirical model function approximates the statistical expectation quite well with a smooth characteristic.

The predication quality of the approximated expectation model is evaluated for the given driving cycles using various 
prediction horizons. The prediction error, expressed as the maximum difference between the actual and predicted propulsion energy, increases to $50 \%$ of the energy storage capacity $\left(\bar{E}_{r}\right)$ for a prediction horizon of 20 time instants (seconds), which can be considered as an upper bound for the prediction horizon in the energy controller. Such prediction horizon is often sufficient to predict the relevant dynamics of relatively small energy buffers such as flywheels.

\section{Controller Design}

A real-time controller is designed following the numerical optimal control-based design approach as described in Section I-A. The optimal control problem is to minimize the overall fuel consumption (chemical energy $E_{f}$ ) for a given driving cycle with initial time instant $k_{0}$ and final time instant $k_{n}$, where $P_{f}$ denotes the fuel power (chemical energy flux), given by

$$
\min _{u} \sum_{k=k_{0}}^{k_{n}-1}\left\{P_{f}(x(k), u(k), w(k)) \Delta t\right\}
$$

subject to the system's dynamics (1) and constraints (2)-(6). There is no final state constraint on $E_{r}$ for energy sustenance, as the flywheel usually contains no energy at the start of a road trip. Nevertheless, the final energy level is often nonzero due to brake energy recuperation and energy state constraints.

\section{A. Optimal Controller}

From previous research, it follows that the optimal control problem can be substantially reduced with only a minor impact on the solution, by neglecting the powertrain temperature $\vartheta_{p}$ from the state space [6], and by considering only power splits $(\sigma)$ that are essential for the functionality of hybrid driving [1] in the control space. These functionalities are: 1) flywheel coasting (no energizing) without disengaging the flywheel clutch to maintain control flexibility $(\sigma=0)$; 2) efficient flywheel energizing $\left(\sigma=\sigma^{*}\right)$, where $\sigma^{*}$ is determined by the statically optimized engine torque $\tau_{e}^{*}\left(\omega_{e}\right)=$ $\arg \min _{\tau_{\mathrm{e}}} P_{f}\left(\omega_{e}, \tau_{e}\right) / \tau_{e} \omega_{e}$, for the engine speed $\left(\omega_{e}\right)$ given by the flywheel speed (or, energy $E_{r}$ ). The reduced optimal control problem can be efficiently solved using DP.

\section{B. Causal Controller}

The controller is made causal using the prediction model (7)-(9) in the MPC framework. This design step is important to gain insights into the main optimal control decisions for a wide range of averaged driving conditions $(w)$ and operating conditions $(x)$. The prediction horizon $k_{z}$ must be short to keep the prediction error small (i.e., $<20$ ) as discussed in Section III-C. However, the horizon must be sufficiently long to include the relevant powertrain dynamics, such that mode switch costs are spread over the driving mode duration, and forced mode switches in the near future (due to constraints) are considered. Using the optimal controller (DP), the average time spent in a driving mode for the relevant urban, nonstandstill and nonbraking parts of a given driving cycle,

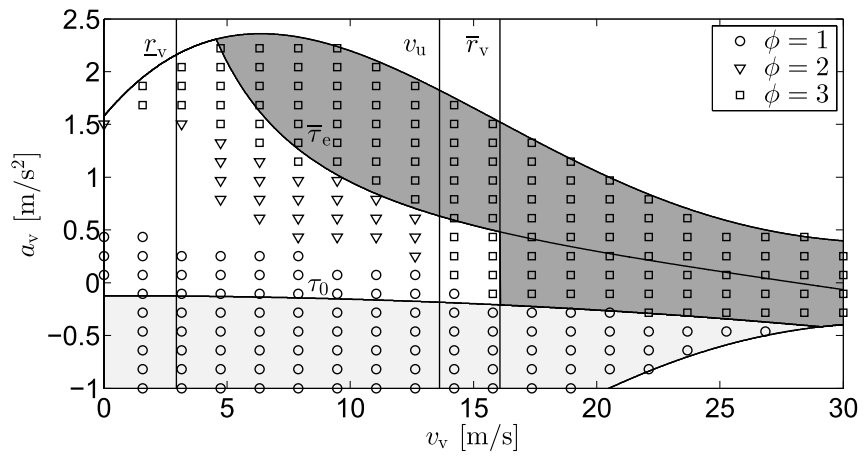

Fig. 3. Control decisions obtained with MPC (symbols) as a function of the driving conditions $\left(v_{v}\right.$ and $\left.a_{v}\right)$, for a given relative flywheel energy of $E_{r} / \bar{E}_{r}=17 \%$ and driving mode $\phi_{\text {pre }}=1$. Control variables that change within three time instants are not shown. The lines denote constraints, whereas the gray areas indicate control regions that are determined by the constraints.

ranges between 7.5 (EHV) and 11.2 (NEDC) time instants. Consequently, a prediction horizon of $k_{z}=15$ is selected.

A part of the resulting controller is shown in Fig. 3 as a function of the driving cycle states $\left(v_{v}, a_{v}\right)$, for given powertrain states $\left(E_{r} / \bar{E}_{r}=17 \%, \phi_{\text {pre }}=1\right)$. Because of the smoothed prediction model, several control regions can be clearly distinguished. However, still some nonsmooth irregularities are observed, which are due to momentary costs caused by the switched dynamics and a finite prediction horizon. In general, the following control decisions are observed: for high power demands $\left(v_{v} \gg 0, a_{v} \gg 0\right)$, the engine is efficiently operated in the engine mode. For low and negative power demands, the flywheel is used for propulsion and braking. For intermediate power demands, both the flywheel and engine are engaged in the hybrid mode. More insights and limitations of this controller are described next.

\section{Real-Time Controller}

Rules can be extracted from the causal controller, which is a difficult process that combines data interpretation with physical understanding of the hybrid powertrain. In the following, two rule types are described to compose the rulebased controller (RB), for which one calibration parameter is assigned that increases its robustness against prediction uncertainties.

1) Feasibility Rules: The feasibility rules restrict the control space to its feasible domain, which are determined by the operating limits of the hybrid powertrain (2)-(6) and shown with lines in Fig. 3. In some regions of the state space, the control space is restricted to only one possible control decision, as indicated by gray areas. The relatively large gray areas reflect the high constraint activity of this powertrain. The remainder (white area) requires additional rules to make a distinct control decision. Although most of the feasibility rules are fixed by the physical powertrain characteristics, a small safety margin may be required for robustness against sensor and estimation uncertainty.

2) Optimality Rules: The optimality rules control the constrained energy dynamics to minimize the fuel consumption for the predicted driving conditions. Two sets of rules 

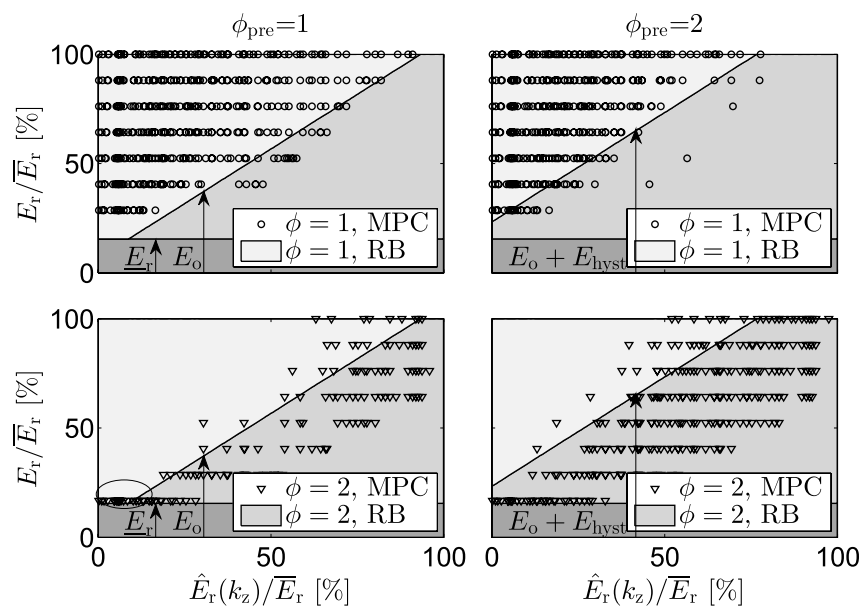

Fig. 4. Control decisions obtained with MPC (symbols) and the energy balance of the RB controller (diagonal line) as a function of the predicted flywheel propulsion energy $\hat{E}_{r}$ and the available flywheel energy $E_{r}$.

TABLE II

RULE PARAMETERs

\begin{tabular}{lrrr}
\hline Parameters & $\hat{k}_{\mathrm{z}}[\mathrm{s}]$ & $E_{\mathrm{o}}[\mathrm{kJ}]$ & $E_{\text {hyst }}[\mathrm{kJ}]$ \\
\hline Fit with MPC controller & 5 & 10 & 25 \\
Calibration with RB controller & 5 & 10 & 50 \\
\hline
\end{tabular}

are distinguished: one set to choose the driving mode $\phi$, and one set to choose the relative power split $\sigma$. The power split rules make the decision between flywheel coasting $(\sigma=0)$ and flywheel energizing $(\sigma=\sigma *)$, and is based on the observation that energizing is only effective when: 1) the flywheel energy level is low; 2) the engine is operated at an inefficient low torque otherwise; and 3) the transmission clutch $\left(C_{t}\right)$ is not slipping, that is, to limit the power dissipation. These rules are described by (13) next.

The driving mode rules make the decision between flywheel driving $(\phi=1)$ and hybrid driving $(\phi=2)$, whereas the irreversible switch to engine driving $(\phi=3)$ is avoided whenever that is feasible. The rules are based on the predicted flywheel energy $\hat{E}_{r}$ described by

$$
\hat{E}_{r}\left(k_{z}\right)=\sum_{k=k_{0}+1}^{\hat{k}_{z}}\left\{\left.f_{1}(x(k), u(k), \hat{w}(k)) \Delta t\right|_{\phi=1}\right\}
$$

where $f_{1}$ is the part of function $f$ in (1) that describes the dynamics of $E_{r}$. The energy balance $E_{r}=\hat{E}_{r}+E_{o}+E_{\mathrm{hyst}}$ is introduced to decide whether sufficient energy is available for flywheel driving $(\phi=1)$ or not $(\phi=2)$. Here, an offset $E_{o}$ and hysteresis band $E_{\text {hyst }}$ are introduced for fitting purposes. Fig. 4 shows the control decisions obtained with MPC (symbols) and the fitted energy balance (diagonal line) as a function of the predicted propulsion energy $\left(\hat{E}_{r}\right)$ and the available flywheel energy $\left(E_{r}\right)$. Each row shows a different control decision $(\phi)$, whereas each column shows a different driving mode state $\left(\phi_{\text {pre }}\right)$. It can be seen that the energy balance separates the control decisions quite well, using the fitted parameters as listed in Table II. Here, $E_{o}$ determines the vertical offset, whereas $\hat{k}_{z}$ determines (implicitly) the slope of the line. The hysteresis band $E_{\text {hyst }}$ gives different energy balances for each driving mode state to damp the switches between driving modes. The mismatch on the left bottom of Fig. 4 can be avoided with an additional rule: the controller must switch to hybrid driving $(\phi=2)$ when the predicted propulsion energy for the next time step [i.e., $\left.\hat{E}_{r}(1)\right]$ drops below the effective energy left in the flywheel (i.e., $E_{r}-\underline{E}_{r}$ ). These rules are described by (12) next.

3) Rule-Based Controller: The combined feasibility and optimality rules, where the priority of each rule is mainly determined by feasibility, are given by

$$
\begin{aligned}
& \phi= \begin{cases}3 & \text { if } E_{r} \leq \underline{E}_{r} \\
1 & \text { else if } \tau_{p}^{\prime} \leq \tau_{0} \\
3 & \text { else if } \phi_{\text {pre }}=3 \vee r_{v}^{\prime} \geq \bar{r}_{v} \vee \tau_{p}^{\prime}>\bar{\tau}_{e} \\
1 & \text { else if } E_{r}>\hat{E}_{r}(1)+\underline{E}_{r} \\
& \wedge\left(\left(\phi_{\text {pre }}=1 \wedge E_{r}>\hat{E}_{r}\left(k_{z}\right)+E_{o}\right)\right. \\
& \left.\vee\left(\phi_{\text {pre }}=2 \wedge E_{r}>\hat{E}_{r}\left(k_{z}\right)+E_{o}+E_{\mathrm{hyst}}\right)\right) \\
3 & \text { else if } \phi_{\text {pre }}=1 \wedge v_{v}>v_{u} \\
2 & \text { else }\end{cases} \\
& \sigma= \begin{cases}\sigma^{*} & \text { if } r_{v}^{\prime} \geq \underline{r}_{v} \wedge \tau_{p} \leq \frac{\bar{E}_{r}-E_{r}}{\bar{E}_{r}-\underline{E}_{r}} \tau_{e}^{*} . \\
0 & \text { else }\end{cases}
\end{aligned}
$$

Here, $r_{v}^{\prime}=\left\{r_{v} \mid \phi=1\right\}$ and $\tau_{p}^{\prime}=\left\{\tau_{p} \mid \phi=1\right\}$ denote, respectively, the speed ratio and the primary torque for a (virtually) engaged flywheel clutch. The associated control regions are shown in Fig. 5(a) and (b) by the gray areas, together with the control regions obtained with MPC (symbols). As expected, the flywheel driving region increases with the flywheel energy at the cost of the hybrid driving region. The hysteresis band follows from the difference between the two figures (i.e., driving mode states). Overall, it can be seen that the RB controller approximate the control regions obtained with MPC quite well with a smooth characteristic.

4) Calibration Parameter: Besides the substantial simplification, the RB controller can be calibrated relatively easily, by using only one calibration parameter: the hysteresis band $E_{\text {hyst }}$. The damping owing to the hysteresis band is required to limit the energy cost of engine cranking, when switching from flywheel driving to hybrid driving. Besides the fuel benefit, the hysteresis band also contributes to a more consistent powertrain response. However, a large value increases the average flywheel energy and therewith the operating speeds of the powertrain components and the associated frictional energy dissipation, so there exists a tradeoff. Using MPC, this tradeoff is found by operating the flywheel energy very close to its minimum $\left(\underline{E}_{r}\right)$, where unnecessary driving mode switches are precisely avoided for the predicted driving cycle. This strategy works well if the actual driving conditions match with the prediction, but unnecessary driving mode switches (i.e., to engine driving) are unavoidable otherwise. This high sensitivity against prediction uncertainties can be reduced by selecting a higher hysteresis band such that the flywheel energy is operated further from its minimum. As a result, the hysteresis band serves as useful calibration parameter to increase 


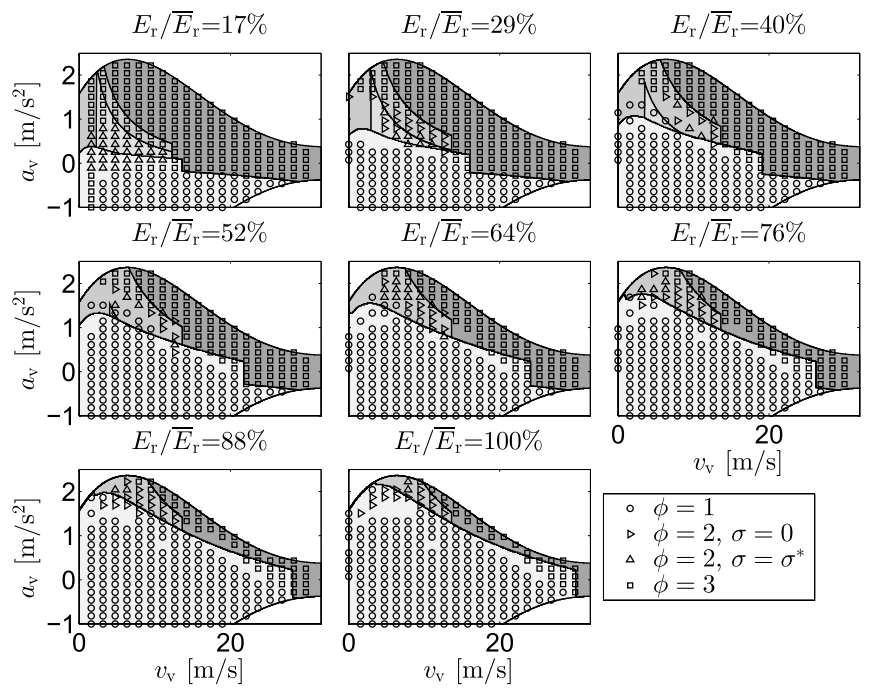

(a) $\phi_{\text {pre }}=1$.

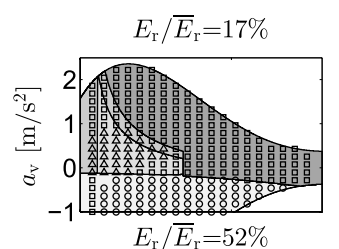

$E_{\mathrm{r}} / \bar{E}_{\mathrm{r}}=29 \%$

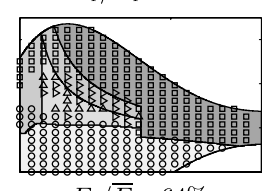

$E_{\mathrm{r}} / \bar{E}_{\mathrm{r}}=64 \%$

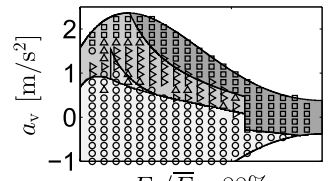

$E_{\mathrm{r}} / \bar{E}_{\mathrm{r}}=88 \%$
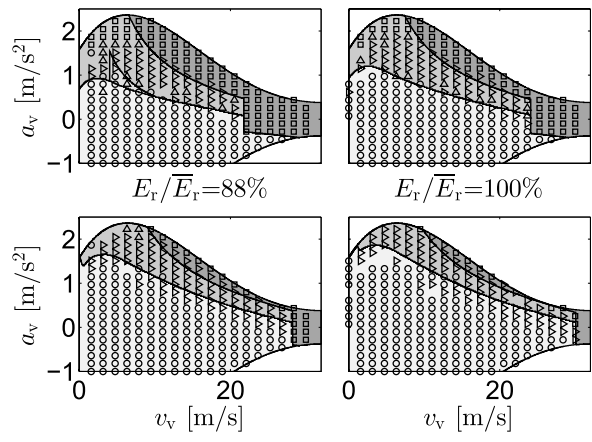

$E_{\mathrm{r}} / \bar{E}_{\mathrm{r}}=100 \%$

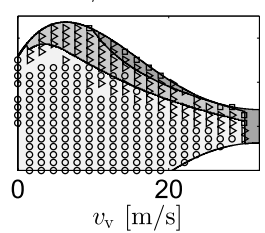

(b) $\phi_{\text {pre }}=2$.

Fig. 5. Control decisions obtained with MPC (symbols) and RB (gray areas) as a function of the vehicle velocity $v_{v}$, acceleration $a_{v}$, flywheel energy $E_{r}$, and the driving mode state $\phi_{\text {pree }}$.

robustness, but at the cost of a higher fuel consumption. The hysteresis band is optimized with respect to the fuel consumption for the six considered driving cycles, resulting in the rule parameters as listed in Table II. The tradeoff between fuel consumption and the number of driving mode switches (consistency), however, remains subjective and should be calibrated in the vehicle.

\section{RESUlts AND Discussion}

Simulations are performed to evaluate the performance of the real-time (RB), causal (MPC), and optimal (DP) energy controllers for different driving cycles and start conditions.

\section{A. Fuel-Saving Potential}

The control performance is expressed as the fuel saving of the mechanical hybrid powertrain with respect to its

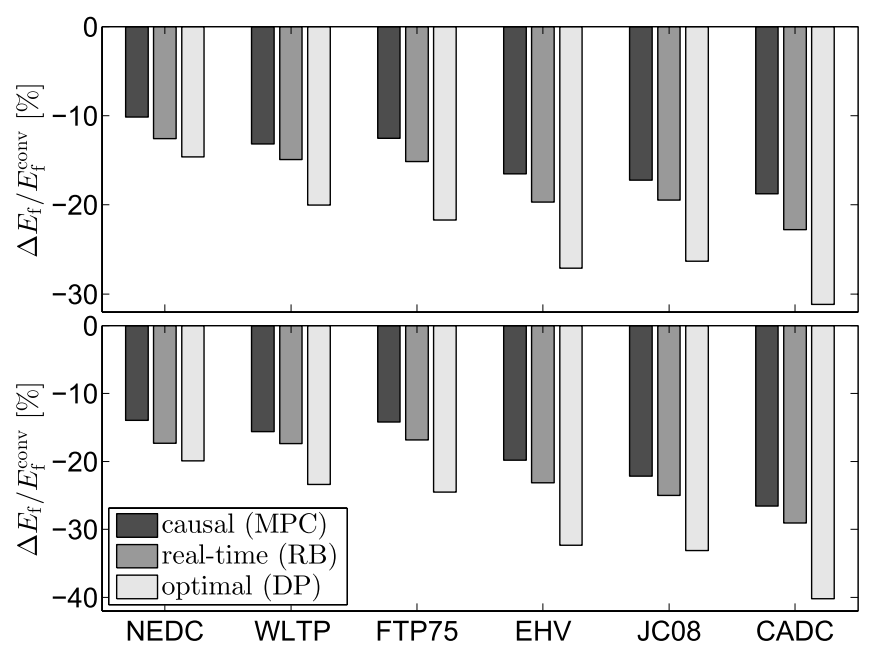

Fig. 6. Fuel-saving potential of the hybrid powertrain with respect to the conventional powertrain using the optimal (DP), causal (MPC), and realtime (RB) controller, for cold start conditions (top graph) and warm start conditions (bottom graph). The fuel-saving potential is computed as the difference in the overall fuel consumption $\left(\Delta E_{f}\right)$ with respect to the overall fuel consumption of the conventional powertrain $\left(E_{f}^{\text {conv }}\right)$.

conventional counterpart without the flywheel system. This fuel-saving potential is computed for cold start conditions, where the powertrain is at its ambient temperature $\left(\vartheta_{p}\left(k_{0}\right)=20{ }^{\circ} \mathrm{C}\right)$ and the flywheel is stationary $\left(E_{r}\left(k_{0}\right)=\right.$ $0 \mathrm{~kJ}$ ), and for warm start conditions, where the powertrain is still at operating temperature $\left(\vartheta_{p}\left(k_{0}\right)=80{ }^{\circ} \mathrm{C}\right)$ and the flywheel contains $50 \%$ of its energy storage capacity $\left(E_{r}\left(k_{0}\right)=\bar{E}_{r} / 2=75 \mathrm{~kJ}\right)$. Fig. 6 shows the computed fuel-saving potential for the three controllers. It can be seen that the RB controller performs consistently better than the MPC controller because of the increased robustness against prediction uncertainties with the optimized calibration parameter, despite the (little) uncertainty added with rule extraction. The fuel-saving potential of the RB is unavoidably lower than that of the optimal DP controller, yet still high for the urban driving cycles (EHV, JC08, CADC) under both warm start conditions $(23.1 \%-29.1 \%)$ and cold start conditions $(19.5 \%-22.8 \%)$. For the mixed driving cycles, the fuel-saving potential is somewhat lower owing to the extra-urban parts where the flywheel system is not used, but still significant for both warm start conditions $(16.8 \%-17.4 \%)$ and cold start conditions $(12.6 \%-15.1 \%)$.

\section{B. Energy Controller}

Detailed results of the energy controller and the powertrain dynamics are shown in Fig. 7(a) for the mild NEDC and in Fig. 7(b) for the sportive EHV. It can be seen that the RB and DP controllers make similar control decisions $(\phi, \sigma)$, whereas the MPC controller makes more often the irreversible switch to engine driving. The good resemblance of the RB and DP controllers implies that the main control decisions are well captured in the MPC controller, as this has formed the structure of the RB controller. The difference between the MPC and RB controllers, however, also shows that cal- 

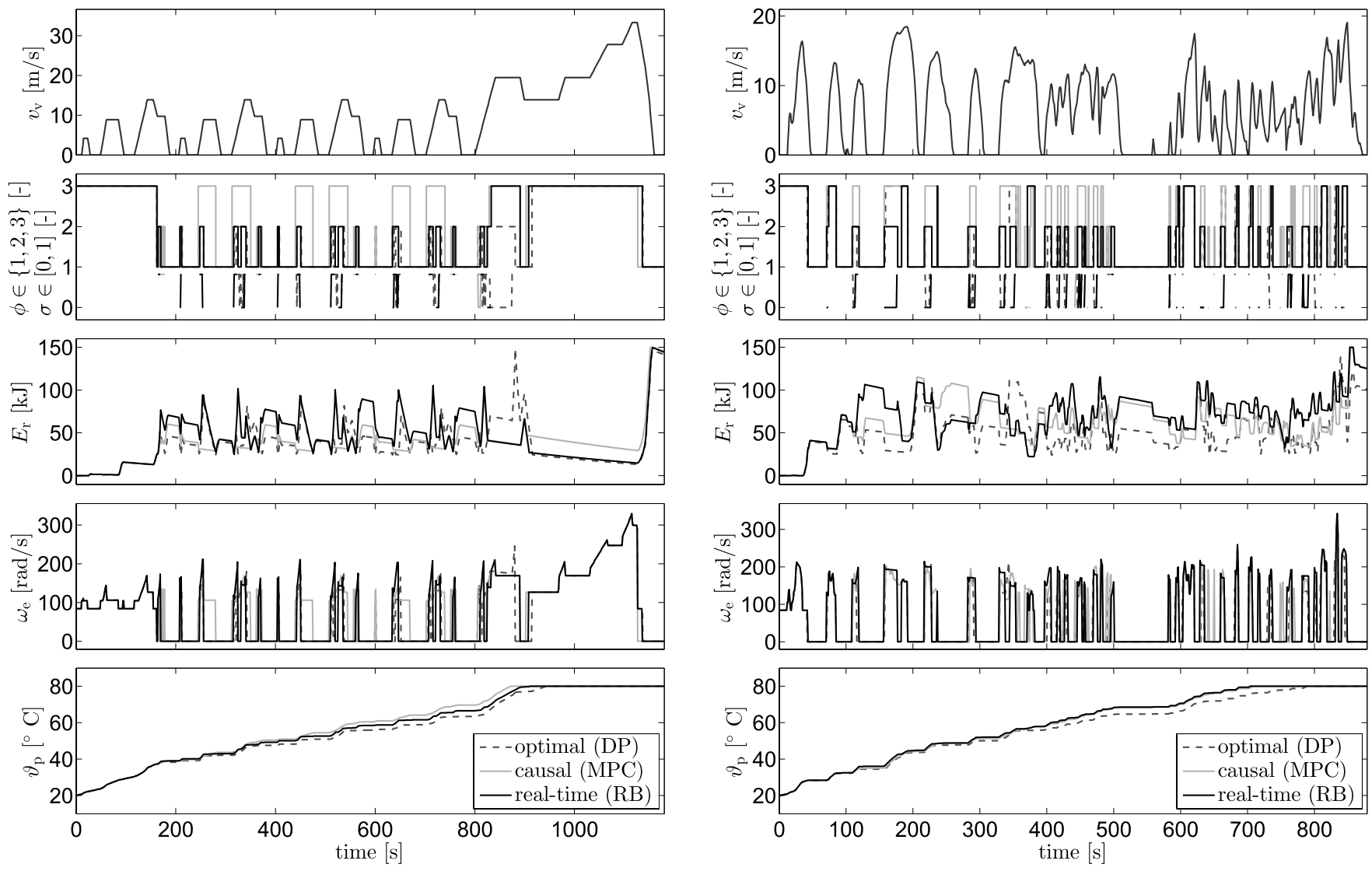

(a)

(b)

Fig. 7. Energy controller and powertrain dynamics with cold start conditions using the real-time controller (RB) and the optimal controller (DP). (a) NEDC. (b) EHV. Top to bottom: velocity of the vehicle $\left(v_{v}\right)$, driving mode $(\phi)$ and power split $(\sigma)$, flywheel energy $\left(E_{r}\right)$, engine speed $\left(\omega_{e}\right)$, and powertrain temperature $\left(\vartheta_{p}\right)$.

ibration of the hysteresis band is crucial to be less sensitive to prediction uncertainties. For the RB and DP controllers, small differences in the control decisions become visible in the flywheel energy $\left(E_{r}\right)$, which is, on average, operated at slightly higher levels than with the optimal controller due to the relatively large hysteresis band. Consequently, the frictional losses increase, which results in a slightly faster heating of the powertrain $\left(\vartheta_{p}\right)$, but also a higher fuel consumption.

\section{CONCLUSION}

This brief has presented the design of a real-time implementable energy controller for a mechanical hybrid powertrain that is based on optimal control. The future driving conditions are modeled by a smooth approximation of statistical data, and implemented in the receding model predictive control framework. The controller is made tunable by rule extraction according to physical understanding of the system, which gives insights in the implicit decision process of the numerical optimization. Using the gained insights, the hysteresis band that damps the switches between flywheel driving and hybrid driving, is assigned as the single calibration parameter to increase the robustness against prediction uncertainties. Results show that, due to the calibration, the rule-based controller outperforms the model predictive controller in terms of fuel saving. The fuel-saving potential with the rule-based controller is inherently lower than with the optimal controller, yet a transparant, causal, and easily tunable controller is obtained that is suitable for real-time implementation.

\section{REFERENCES}

[1] K. van Berkel, T. Hofman, B. Vroemen, and M. Steinbuch, "Optimal control of a mechanical hybrid powertrain," IEEE Trans. Veh. Technol., vol. 61, no. 2, pp. 485-497, Feb. 2012.

[2] K. van Berkel, S. Rullens, T. Hofman, B. Vroemen, and M. Steinbuch, "Topology and flywheel size optimization for mechanical hybrid powertrains," IEEE Trans. Veh. Technol., doi: 10.1109/TVT.2014.2312646.

[3] K. van Berkel, F. Veldpaus, T. Hofman, B. Vroemen, and M. Steinbuch, "Fast and smooth clutch engagement control for a mechanical hybrid powertrain," IEEE Trans. Control Syst. Technol., vol. 22, no. 4, pp. 1241-1254, Jul. 2014.

[4] U. Christen and R. Busch, "The art of control engineering: Science meets industrial reality," in Proc. Int. Fed. Autom. Control (IFAC) ECOSM, Rueil-Malmaison, France, 2012.

[5] A. Sciarretta and L. Guzzella, "Control of hybrid electric vehicles," IEEE Control Syst., vol. 27, no. 2, pp. 60-70, Apr. 2007.

[6] K. van Berkel, W. Klemm, T. Hofman, B. Vroemen, and M. Steinbuch, "Optimal control of a mechanical hybrid powertrain with cold-start conditions," IEEE Trans. Veh. Technol., vol. 63, no. 4, pp. 1555-1566, May 2014.

[7] G. Paganelli, G. Ercole, A. Brahma, Y. Guezennec, and G. Rizzoni, "General supervisory control policy for the energy optimization of charge-sustaining hybrid electric vehicles," JSAE Rev., vol. 22, pp. 511-518, Oct. 2001.

[8] J.-S. Won, R. Langari, and M. Ehsani, "An energy management and charge sustaining strategy for a parallel hybrid vehicle with CVT," IEEE Trans. Control Syst. Technol., vol. 13, no. 2, pp. 313-320, Mar. 2005. 
[9] Y. Zhu, Y. Chen, Z. Wu, and A. Wang, "Optimisation design of an energy management strategy for hybrid vehicles," Int. J. Alternat. Propulsion, vol. 1 , no. 1 , pp. 47-62, 2006.

[10] J. T. B. A. Kessels, M. W. T. Koot, P. P. J. van den Bosch, and D. B. Kok, "Online energy management for hybrid electric vehicles," IEEE Trans. Veh. Technol., vol. 57, no. 6, pp. 3428-3440, Nov. 2008.

[11] S. Delprat, J. Lauber, T. M. Guerra, and J. Rimaux, "Control of a parallel hybrid powertrain: Optimal control," IEEE Trans. Veh. Technol., vol. 53, no. 3, pp. 872-881, May 2004.

[12] D. Ambühl, O. Sundström, A. Sciarreta, and L. Guzzella, "Explicit optimal control policy and its practical application for hybrid electric powertrains," Control Eng. Pract., vol. 18, no. 12, pp. 1429-1439, 2010.

[13] E. D. Tate, J. W. Grizzle, and H. Peng, "Shortest path stochastic control for hybrid electric vehicles," Int. J. Robust Nonlinear Control, vol. 18, no. 14, pp. 1409-1429, 2007.

[14] D. F. Opila, X. Wang, R. McGee, R. B. Gillespie, J. A. Cook, and J. W. Grizzle, "An energy management controller to optimally trade off fuel economy and drivability for hybrid vehicles," IEEE Trans. Control Syst. Technol., vol. 20, no. 6, pp. 1490-1505, Nov. 2012.
[15] M. Koot, J. T. B. A. Kessels, B. de Jager, W. P. M. H. Heemels, P. P. J. van den Bosch, and M. Steinbuch, "Energy management strategies for vehicular electric power systems," IEEE Trans. Veh. Technol., vol. 54, no. 3, pp. 771-782, May 2005.

[16] H. Borhan, A. Vahidi, A. M. Phillips, M. L. Kuang, I. V. Kolmanovsky, and S. Di Cairano, "MPC-based energy management of a power-split hybrid electric vehicle," IEEE Trans. Control Syst. Technol., vol. 20 , no. 3, pp. 593-603, May 2012.

[17] C.-C. Lin, H. Peng, J. W. Grizzle, and J.-M. Kang, "Power management strategy for a parallel hybrid electric truck," IEEE Trans. Control Syst. Technol., vol. 11, no. 6, pp. 839-849, Nov. 2003.

[18] B. Glaser and A. Strauss, The Discovery of Grounded Theory: Strategies for Qualitative Research, 7th ed. Piscataway, NJ, USA: Transaction Publishers, 2012.

[19] K. van Berkel, M. Maessen, T. Hofman, B. Vroemen, and M. Steinbuch, "Semi-empirical power dissipation modelling of mechanical hybrid powertrain components," Proc. IMechE, J. Automobile Eng., vol. 228, no. 4, pp. 443-456, 2014. 